\title{
The Application of Multimedia Database in the Network Teaching of University Sports Elective Course
}

\author{
Guo sheng \\ P.E.College , Beihua University \\ Jilin City, Jilin Province, China
}

Keywords: Multimedia database, Distributed database, Internet, Client/server structure.

\begin{abstract}
Network teaching based on computer has been a new teaching mode put forward in recent years. The design and organization of multimedia database is the foundation of the whole network construction and the important standards for network teaching quality evaluation. The network transmission bandwidth is limited, which makes a higher request for database redundancy, calculating method of inquiry and structure distribution. This paper makes specific instructions for the system structure and construction method of the application of multimedia database in the network teaching of university sports elective course.
\end{abstract}

\section{Introduction}

To realize information exchange and resource sharing between the computer and the computer, the computer network is made up of communication lines. According to the computer network coverage, it can be divided into LAN, Intra city networks and the Wan. In order to realize the information transmission within larger scope, more computer networks can be connected, which constitutes the Internet, a kind of nets between nets. It is a universal network through protocol TCP/IP. Multimedia database design is the key of network teaching. Multimedia question bank, adaptive test and expert system and so on belong to the application of multimedia database in the network teaching of the university sports elective course.

\section{The present model of network database}

So far, there are two kinds of the extensive use of database present method, one kind is a web page mode, such as ASP, the web site register and login function of which is most common; the other is window mode, which uses window interface to present data, such as ICQ (your buddy list is stored in the distant database server), super star reader, etc. The characteristics are as follows: the database distribution structure is Clint/Server model, that is data stored in the data server. The local machine do not save any data relevant to database. The server and client share data in "request and response" way. The result subsets transmitted in the form of flow can be inquired from server in asynchronous methods. Having been operated in the client, data sets will not be preserved for good. The essential differences between web page mode and window mode lie in that because of restrictions on network security, the database based on the browser way can't operate local files ( cooking except ), while the database based on window mode can make necessary operation on the resources of the local machine. But the former telecommunications can be connected by browser automatically and the latter must be connected by the programmer adjusting WINSOCK API. This shows that it is not necessary to install client software based on the browser's way, but which limits resource's operation to a great degree. The client resource's operation based on window way has large flexibility, but the design of which is very complicated in data connection of the stability and the safety aspects. 


\section{Advancing of new database structure}

Now there are two network teaching database frameworks. I will propose a new database structure---master-slave distributed model. We put the data closely related to the client in the local and store the data related to the server in the distance, thus forming the distribution of processing model. The following example: there is a Student test check grade system, and its database consists of two dimensional tables including students' basic information and test scores, which is associated by the Student Number. If using Clint/Server structure, we have to use the SQL statement to inquire the result subsets after the two tables. subsets. If putting the Student in the client list, we can get the result subsets only using Selecting Where Student $\backslash$ Number $=$ Mark, thus greatly reducing the amount of data transmission between networks. After testing in the browser based on ASP environment, a record (in article 154 records in search) including 14 columns from the local machine to returning needs $5 \sim 7$ seconds, therefore to reduce the number of field returning is an effective method to reduce inquire time. The database model will expand the traditional relational model to the remote connection relationship, as the following chart shows:

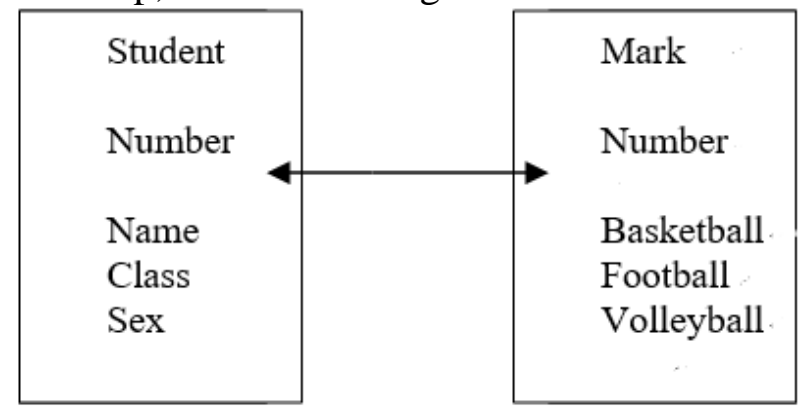

Figure 1. Distribution model of database

\section{Transformation from relationship model to semantic model}

The model for the modern database is mostly relation model, whose essence is the database structure model expanded from e-r model . Semantic model is put forward in 1988 that is based on the semantic objects and properties, the building structure of which is similar to the OOP method. As the chart shows is the semantic model database views:

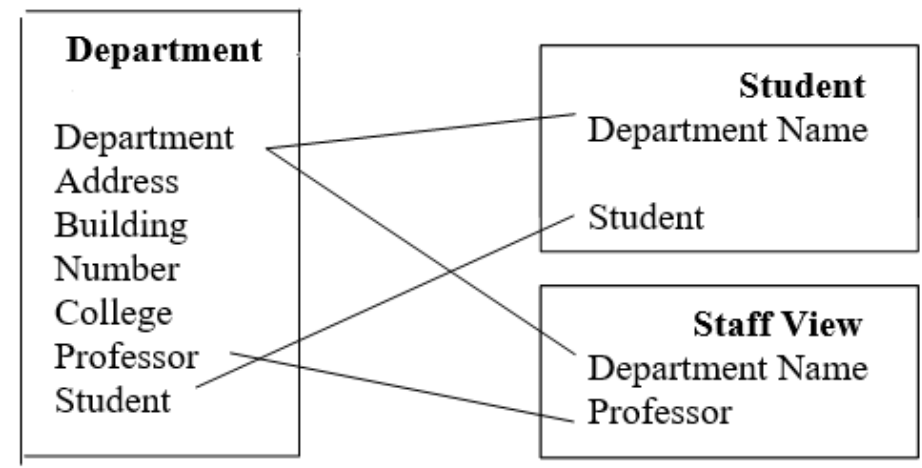

Figure 2. Semantic model object department

"The Department" attributes in two application views are visible. But only in the Student List view Student is showed, and PROFESSOR is visible only in the Staff. So the Department is the parent-class, and PROFESSOR and Student is its private property, the scope of them being Student List and Staff tables. Semantic model, compared to relationship model , the biggest advantage is that when inquiring a database, we only need a part, not all fields (called attributes in the semantic model), but the relationship model can only record the minimum inquire unit. Semantic model shortens the inquire time through shielding some properties, such as in the above table, not viewing the PROFESSOR in Student List, and reduces the number of inquire field to improve the response capacity of the data platform in the network teaching of the university sports elective course. 


\section{The application of fuzzy database}

From the concept of database put forward to now, dozens of kinds of data models has been put forward, but almost all of the data models only show accurate data, and the effect of fuzzy data said is very poor. As in students' achievement database of middle school students, math is 75 points (accurate data), but you can't assess which grad is excellent, good and bad etc, and this is fuzzy data. If you think 75 is good, what about 74 points? Good or Moderate? In the teaching, there are a lot of fuzzy problems like that, which are introduced into the concept of fuzzy data model. Set D1, D2, ...... . Dn for several domains, relationship R (D1, D2,..... Dn) for cross product, D1 $X \backslash$ D2...... A subset of the $X \mathrm{Dn}$, and set the containing expressed as $\mathrm{P}(\mathrm{X} 1 \mathrm{X} 2, \ldots \ldots, \mathrm{Xn}), \mathrm{Xi} \in \mathrm{Di}, \mathrm{I}=1$, $2, \ldots . .$. , n. If some of them, without constraint, will be changed into a fixed value in the corresponding field, for example, X2, X3,..... , Xn are constrained into d2, d3,..... , dn (di for constant of D1), we receive limited predicate P (X1, d2,..... dn), thus Xi and R (D1, d2,..... dn) form a "tuple function" noted for (X1, d2,..... dn), X1 $\in$ di, Set fuzzy rules P1 (x, y), P2 (y, z)-Q (x, z), $\mathrm{CF}$, variables of the domain are respectively $\mathrm{x} \in \mathrm{x}, \mathrm{y} \in \mathrm{y}$ and $\mathrm{z} \in \mathrm{z}$; expressing tuple function: (P1, $\mathrm{x}, \mathrm{y}, \mathrm{z}, \mathrm{P} 2, \mathrm{y}, \mathrm{Q}, \mathrm{x}, \mathrm{z}, \mathrm{CF}$, ?)

There is a semantic example, "if X's achievement is better than Zhang San's and Zhang San's is better than Y's, we know X's is better than Y's, which can be shown in the following tuple function(achievement, X, Zhang San, achievement, Zhang San, Y, achievement, X, Y, achievement, 1)

Among them, the $\mathrm{X}$ and $\mathrm{Y}$ are changeable tuples. "Zhang San" as a fixed value, shown in a result table (grades and name), firstly according to the first premise to find a student better than Zhang San, again according to the second premise to find another student with poorer grades than Zhang San, which an inquire subset can be formed based on. As a result of the relationship explaining reasoning rules, inquiring taking place of reasoning thinking of the human brain, the teaching data processing ability is greatly improved and it is advantageous to handle complex teaching data.

To sum up, we theoretically analyze several development directions of the multimedia database in the network teaching of the universities sports elective course (that is, the distributed processing, the use of semantic model, fuzzy technology application), and research its feasibility. Because of the complexity of the teaching data and the constraint of network condition, all the three development directions are to enhance improving the speed of inquiring data. Now we give a general E-School solution according to the actual situation.

\section{Visual E-School plan}

Basic requirements: hardware: campus network or teaching LAN; a server; some clients no disk workstation (also adding hard disk); network adapter and Boot Room. Software: WinNT4.0 or Win2000 Server or Win9X, IIS or PWS (Personal Webs)

As shown in figure of virtual teaching net structure:

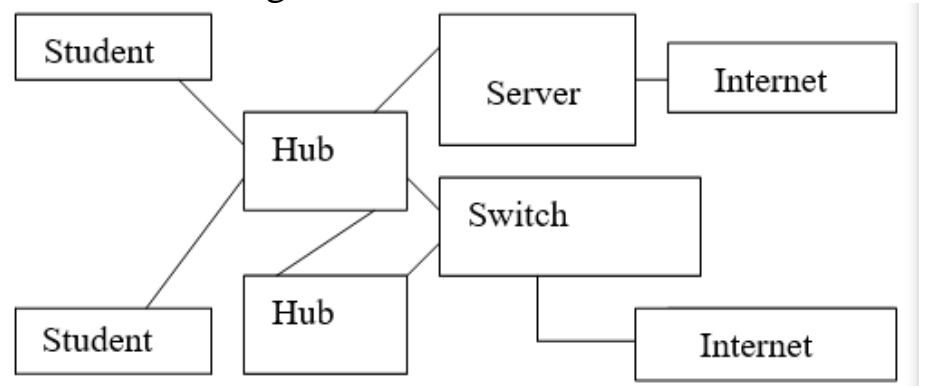

Figure 3. Virtual teaching network connection schemes

With the Access database for data platform, front desk with ASP, the relationship among all the parts finished by the database connection, all the data from the database records, E-School is an online education platform based on the multimedia database. It can replace teachers to complete teaching, assignments, exams, etc. The interactivity and intellect of online teaching is key to judge its quality and which can be realized in the perfect network database system. There is a Visual E-School 
Module as follows:

\section{A. E-School homepage}

\section{B. Students login to homepage(no students in the database)}

Including classroom teaching module; Test module; Experts on the judgment seat; Experience exchange module; Teaching game module; Homework module

\section{Logout}

Among them the "experience exchange" module (chat rooms), "experts on the judgment seat" module (BBS), teachers teaching, feedback instruction and homework uploading etc. form feedback network, whose function system is based on the application of the database. The following figure:

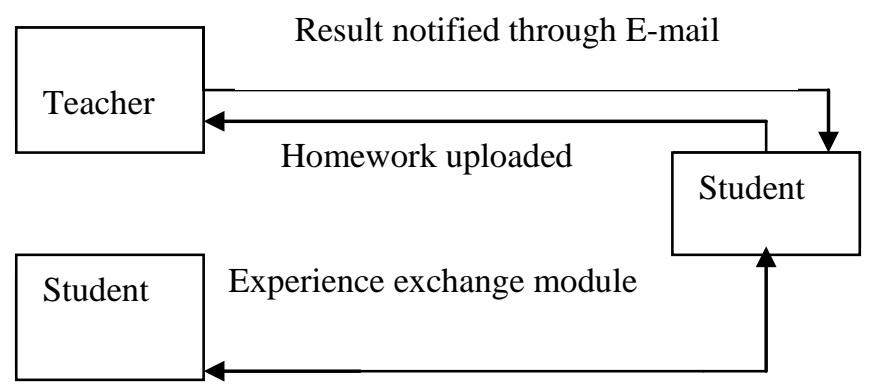

Figure 4. E-School feedback network

To sum up, the application of multimedia database in the network teaching of the university sports elective course is very broad and effective. We can use network database collect data, establish feedback network, semantic networks and even the application of artificial intelligence, thus solving all kinds of problems and difficulties existing in the teaching process, which can't be solved in traditional ways. Only when students can use tools to help themselves to collect information from the outside world, and process information and apply information, can the perfect integration between the information technology and the sports discipline be promoted. It is necessary for students to study and exercise independently with modern scientific technology in order to meet the needs of the lifelong sports.

\section{References}

[1] .Li Hong song, The computer network technology, the publishing house of electronics industry

[2] Database principles, the higher education press

[3] Zhang Jiping and Zhang Qinzhu, Computer and education, the publishing house of electronics industry

[4] Gao Chuanshan,The computer network course, Fu dan university press

[5] He Xin gui, The fuzzy database system, Qinghua university press

[6] David m., The database processing, the publishing house of electronics industry 